\title{
SPRING CENTRAL SECTIONAL SAMPLER
}

\section{Knot Theory and Complex Curves}

\section{Matthew Hedden}

Topology is often intertwined with analysis and geometry in unexpected and beautiful ways. A familiar example of this phenomenon is provided by the Riemann mapping theorem: if the topology of a domain in the complex plane is simple enough, then it is analytically simple. In particular, if it is simply connected and not all of $\mathbb{C}$, then it is holomorphically equivalent to the unit disk. A variant of the mapping theorem can be stated as "a loop in the plane without self-intersections bounds a region that is holomorphically equivalent to the unit disk."

From a topological perspective, loops in $\mathbb{C} \cong \mathbb{R}^{2}$ without self-intersections are quite simple. Indeed, any such loop can be deformed to any other through a family. If one allows loops to extend into $\mathbb{R}^{3}$, the situation becomes far more complicated. Knot theory studies loops in $\mathbb{R}^{3}$ without self-intersections, up to an equivalence defined by smooth deformation. It is convenient to regard loops in $\mathbb{R}^{3}$ as living instead in the 3-dimensional sphere $\mathbb{S}^{3}$ that arises from compactification. This sphere can also be viewed as the set of unit vectors in $\mathbb{C}^{2}$. From this perspective one can ask about an analogue of the Riemann mapping theorem: which knots in the unit sphere $\mathbb{S}^{3} \subset \mathbb{C}^{2}$ bound holomorphically embedded disks in the interior of the unit 4-ball?

It turns out that an answer comes from a solution to a closely related question that will frame my talk at the AMS Spring Central Sectional Meeting. Recall that a complex polynomial in two variables $f(z, w) \in \mathbb{C}[z, w]$ specifies an algebraic curve $V_{f} \subset \mathbb{C}^{2}$ through its zero locus. My talk will address the following question.

Question. Which knots arise as the intersection of an algebraic curve with the unit 3-sphere in $\mathbb{C}^{2}$ ?

I'll call such knots $\mathbb{C}$-knots. The connection between the question above and holomorphically embedded disks comes from the fact that the part of an algebraic curve in the interior of the unit 4-ball can be parametrized by a holomorphic map from a Riemann surface. Because I

Matthew Hedden is a professor of mathematics at Michigan State University. His email address is mhedden@math. msu. edu.

This sampler originally appeared in the April 2020 Notices, but the associated meeting was rescheduled. We reprint it here in advance of the Spring Central Sectional Meeting in March 2022.

For permission to reprint this article, please contact:

reprint-permission@ams.org.

DOI: https://doi.org/10.1090/noti2069

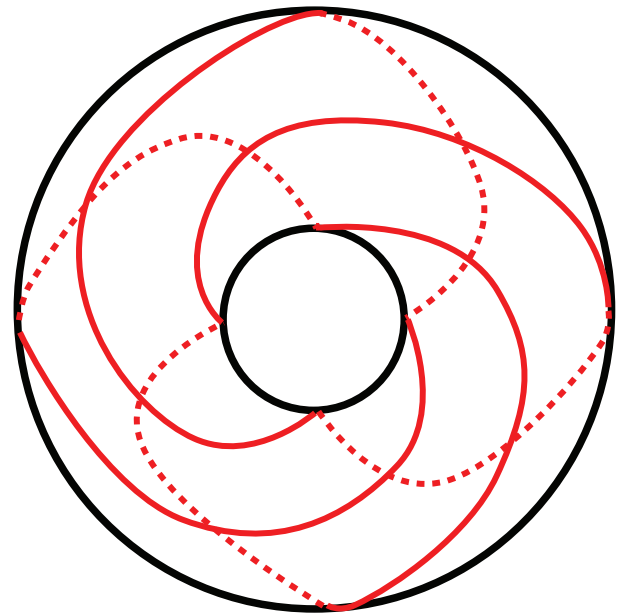

Figure 1. The red curve represents the intersection of the unit 3-sphere $\mathbb{S}^{3} \subset \mathbb{C}^{2}$ with the zero locus of the polynomial $f(z, w)=z^{3}-w^{4}$.

work with knots, which are loops up to deformation, I can smoothly vary the loop in its equivalence class so that the distinction between a holomorphic map and an algebraic curve disappears.

Example. A quintessential example of a $\mathbb{C}$-knot is provided by the polynomial $f(z, w)=z^{3}-w^{4}$. The zero locus of $f$ can be parametrized by the holomorphic map $\gamma: \mathbb{C} \rightarrow \mathbb{C}^{2}$ given by

$$
t \stackrel{\gamma}{\longrightarrow}\left(t^{4}, t^{3}\right)
$$

Considering the intersection of the curve with the 3sphere of vectors $(z, w)$ satisfying $|z|^{2}+|w|^{2}=1$, we obtain the equation $|t|^{8}+|t|^{6}=1$. As the left-hand side is an increasing continuous function of $|t| \geq 0$, we find there is a unique solution $\lambda$. This shows that the intersection between the image of $\gamma$ and the 3-sphere is an embedded circle, parametrized by

$$
\lambda e^{i \theta} \stackrel{\gamma}{\longrightarrow}\left(\lambda^{4} e^{4 i \theta}, \lambda^{3} e^{3 i \theta}\right) .
$$

Geometrically, this is a curve that lies on the torus $\lambda^{4} \mathbb{S}^{1} \times$ $\lambda^{3} \mathbb{S}^{1} \subset \mathbb{C} \times \mathbb{C}$ and that wraps 4 times around the first circular coordinate while wrapping 3 times around the second. The knot represented by this curve is called the torus knot of type $(4,3)$ and is illustrated in Figure 1 . Note that the knot bounds an embedded disk, holomorphically parametrized by the restriction of $\gamma$ to the disk of radius $\lambda$, but that the mapping is singular at the origin where the gradient of $\gamma$ vanishes.

The subject of $\mathbb{C}$-knots lies at the crossroads of a number of branches of mathematics. These knots play an important role in low-dimensional topology, where they have been the subject of guiding conjectures. In algebraic geometry they figure prominently in the study of singular points of algebraic curves (like the origin in the example above). They are tightly connected to certain 
geometric structures on 3-manifolds called contact structures and to analogous symplectic structures on smooth 4manifolds. They are closely related to braid groups and mapping class groups, wherein $\mathbb{C}$-knots admit group theoretic characterizations. A rich interaction has also been witnessed between $\mathbb{C}$-knots and topological invariants stemming from the fields of gauge theory, Floer homology, and categorification. In my talk I will survey the history of $\mathbb{C}$ knots, discuss a number of the interactions mentioned above, and highlight some recent advances and generalizations in their study.

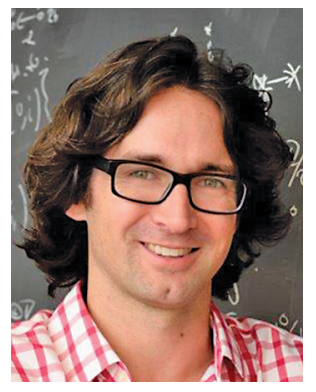

\section{Matthew Hedden}

\section{Credits}

Figure 1 was created by the author.

Photo of Matthew Hedden is by Harley J. Seeley.

\section{FEATURED TITLES FROM

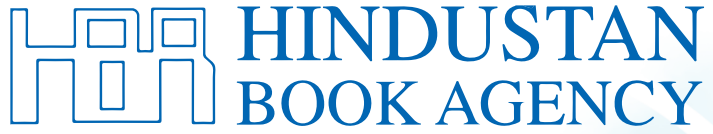

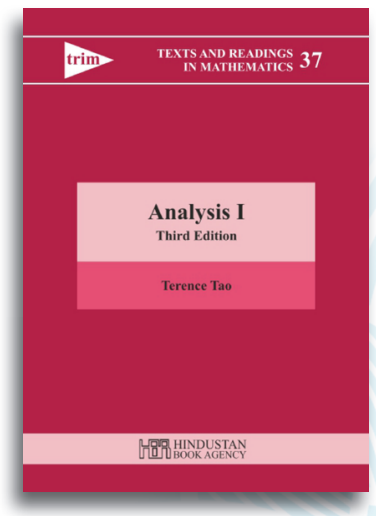

Analysis I

Third Edition

Terence Tao, University of California, Los Angeles,

$C A$

This is part one of a two-volume introduction to real analysis and is intended for honours undergraduates who have already been exposed to calculus. The emphasis is on rigour and on foundations. The

material starts at the very beginning-the construction of the number systems and set theory-then goes on to the basics of analysis (limits, series, continuity, differentiation, Riemann integration), through to power series, several variable calculus and Fourier analysis, and finally to the Lebesgue integral.

Hindustan Book Agency; 2014; 368 pages;

Hardcover; ISBN: 978-93-80250-64-9; List US\$50; AMS members US\$40; Order code HIN/66

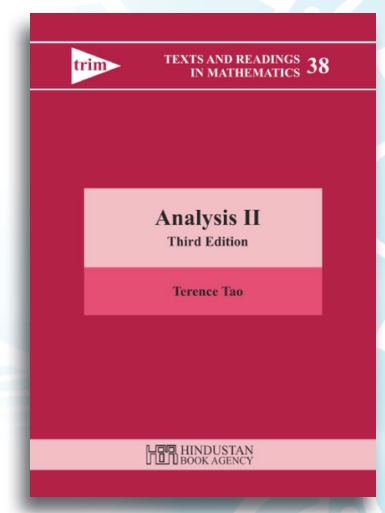

\section{Analysis II Third Edition}

Terence Tao, University of California, Los Angeles

This is part two of a two-volume introduction to real analysis and is intended for honours undergraduates who have already been exposed to calculus. The emphasis is on rigour and on foundations.

Hindustan Book Agency; 2014; 236 pages;

Hardcover; ISBN: 978-93-80250-65-6; List US\$40;

AMS members US\$32; Order code HIN/67

Titles published by the Hindustan Book Agency (New Delhi, India) include studies in advanced mathematics, monographs, lecture notes, and/or conference proceedings on current topics of interest.

Discover more books at bookstore.ams.org/hin.

Publications of Hindustan Book Agency are distributed within the Americas by the American Mathematical Society. Maximum discount of $20 \%$ for commercial channels.

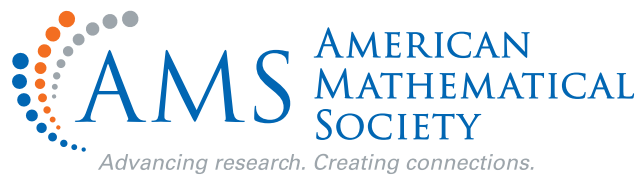

